\title{
Rare case of spontaneous Tumor Lysis Syndrome (TLS) in a patient with Germ Cell Tumor (GCT): literature review and approach to management
}

\begin{abstract}
Tumor Lysis Syndrome (TLS) is an oncologic emergency caused by destruction of malignant cells and release of intracellular components into the blood stream leading to numerous electrolyte abnormalities. Spontaneous TLS in solid tumors, without the presence of precipitating chemotherapy, has rarely been described. Germ cells tumors are the most common malignancy among men age 15-35years of age. Due to the dramatic sensitivity of germ cell tumors to platinum based chemotherapy, systemic treatment in combination with surgical resection lead to cure rates ranging from $50 \%$ $90 \%$. In a patient with spontaneous TLS, prior to initiation of chemotherapy, careful consideration of the treatment regimen is required. Although there are potential adverse effects of chemotherapy in any patient, these side effects may be further exacerbated in patients with significant co-morbidities. We will review a case of metastatic germ cell cancer presenting with spontaneous TLS and discuss implications for management in the acute setting.
\end{abstract}

Volume 7 Issue I - 2017

\section{Joshua Mansour,' Matt Brill, ${ }^{2}$ Theodore Gourdin'}

'Division of Hematology/Oncology, Medical University of South Carolina, USA

${ }^{2}$ Division of Radiology, Medical University of South Carolina, USA

Correspondence: Joshua Mansour, Medical University of South Carolina, USA, Email mansour@musc.edu

Received: July 12, 2017 | Published: July 20, 2017

\section{Introduction}

Tumor Lysis Syndrome (TLS) is an oncologic emergency caused by massive lysis of malignant cells and release of intracellular components into the blood stream. TLS is associated with numerous electrolyte abnormalities including hyperuricemia, hyperkalemia, and hyperphosphatemia. The end result of these metabolic derangements is often acute kidney failure through a variety of mechanisms including uric acid crystal and calcium phosphate crystal deposition in the renal tubules. ${ }^{1}$ TLS usually occurs after starting cytotoxic medications and most commonly after induction chemotherapy in hematologic malignancies. However, spontaneous TLS in solid tumors, without the presence of precipitating chemotherapy, has rarely been described..$^{2-4}$

Germ cells tumors are the most common malignancy among men age 15-35years of age and this incidence has been rising over the past 50years. ${ }^{5,6}$ Due to the dramatic sensitivity of germ cell tumors to platinum based chemotherapy, systemic treatment in combination with surgical resection lead to cure rates ranging from $50 \%$ to $90 \%$, even in metastatic cases. ${ }^{7,8}$ Since the late 1980 s, the regimens of cisplatin and etoposide or bleomycin, etoposide, and cisplatin represent the standard treatment for disseminated germ cell tumors. ${ }^{9}$ In a patient with spontaneous tumor lysis syndrome, prior to initiation of chemotherapy, careful consideration of the treatment regimen is required. Although there are potential adverse effects of chemotherapy in any patient, these side effects may be further exacerbated in patients with significant co-morbidities. We will review a case of metastatic germ cell cancer presenting with spontaneous tumor lysis syndrome and discuss implications for chemotherapeutic management in the acute setting.

\section{Case report}

The patient is a 20 -year-old male who initially noticed swelling in his scrotum for approximately 3-4 months after being hit with a pellet gun in his groin. He was evaluated by a local physician and treated for epididymitis, but after no improvement in symptoms, he presented to the Emergency Department at an outside hospital with worsening scrotal and abdominal pain. A CT abdomen/pelvis was performed and revealed wide-spread metastatic disease to the liver, lungs, and retroperitoneum (Figure 1A) (Figure 1B). He underwent a testicular ultrasound $(\mathrm{U} / \mathrm{S})$, which revealed an enlarged right testicular mass. He subsequently underwent a right-sided orchiectomy and was diagnosed with nonseminomatous testicular cancer (further specifics of pathology were pending at time of initial management). The patient was then discharged home to follow up as an outpatient.

One day after discharge, the patient presented back to the original institution with new abdominal pain, fevers, and chills. He had acute renal failure (Creatinine of 3.0), lactic acidosis (lactate of 3.9), transaminitis (AST of 203, ALT of 126), and hyperbilirubinemia (3.2). He was admitted for presumed septic shock. Serum B-HCG level was 130,000 , AFP was $>11000$, and LDH was $>10000$. Repeat CT abdomen/pelvis revealed stable metastatic disease in the liver, lungs and retroperitoneum as well as new hepatomegaly. Repeat U/S of the scrotum revealed diffuse swelling with a normal left testicle. Renal $\mathrm{U} / \mathrm{S}$ was performed, given his AKI, and appeared to be within normal limits with no obstructive uropathy from metastatic disease. MRI of the brain showed no evidence of acute intracranial abnormalities. Blood cultures were drawn and the patient was started on broad spectrum antibiotics as well as fluid resuscitation. Over the subsequent day, the patient had worsening renal and hepatic failure with a $\mathrm{Cr}$ of 4.8 , INR of 1.8, Alkaline Phosphatase of 639, AST of 203, ALT of 126, and bilirubin of 5 (Table 1). He was transferred to our institution to aid with ongoing management.

On admission to the medical intensive care unit (MICU) at our institution, a uric acid level was drawn and found to be $20.3 \mathrm{mg} / \mathrm{dL}$. The patient received $6 \mathrm{mg}$ IV rasburicase immediately with subsequent uric acid level dropping to $17.9 \mathrm{mg} / \mathrm{dL}$. He was started on IV fluids $(150 \mathrm{mEq} \mathrm{NaHCO} 3$ at $200 \mathrm{~mL} / \mathrm{hr})$ and renally dosed allopurinol at $50 \mathrm{mg}$ orally three times daily. Nephrology was consulted due to concern for acute uric acid nephropathy secondary to spontaneous 
TLS. Creatinine was improving to 3.0 but it was recommended that the patient immediately start hemodialysis and continue it during systemic chemotherapy with anticipation of further electrolyte abnormalities in the context of continued tumor lysis.

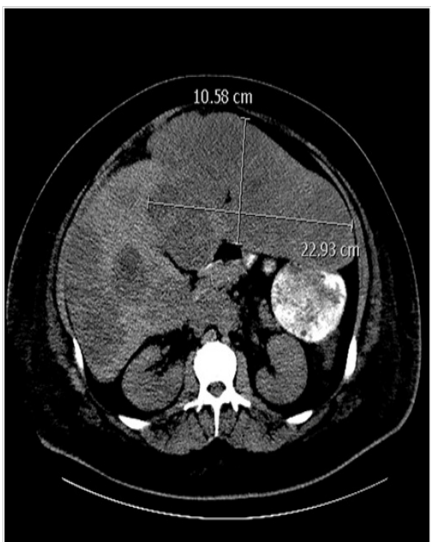

Figure IA (CT without contrast prior to therapy).

Following a session of hemodialysis, we had a lengthy discussion with the patient about the nature of his illness and the risks and benefits of potential chemotherapy. We then proceeded with a cycle of dose attenuated carboplatin and etoposide. Carboplatin was administered at an AUC equivalent of 5 on day one along with etoposide $40 \mathrm{mg} /$ $\mathrm{m}^{2}$ on days one through three. Using the Calvert formula and a GFR of 30 , his renal function between dialysis sessions, we determined the carboplatin dose to be $400 \mathrm{mg}$. He underwent an additional hemodialysis session 15hours following the carboplatin (timing as advised by clinical pharmacy services and data review) to further reduce the risk of renal toxicity. Hemodialysis continued for another session, and after monitoring the patient's renal function for a week, dialysis was ultimately discontinued.

The patient was in the hospital for 15 total days. Spontaneous renal function recovered one week after admission with creatinine continuing to downtrend to 0.9 at discharge. Bilirubin slowly improved with value of 2.0 at discharge. The patient was seen in outpatient follow-up at our institution to continue care one week after discharge (3weeks after initial presentation). Labs at that point showed tumor marker response with serum B-HCG level of 4150, AFP of 1611, and LDH 1187. Creatinine/Bilirubin at that point were 1.0 and 0.9 respectively. BEP therapy was initiated with plan for 4 cycles. To this point, the patient is tolerating this therapy without unexpected toxicity and has had normalization of serum tumor markers.

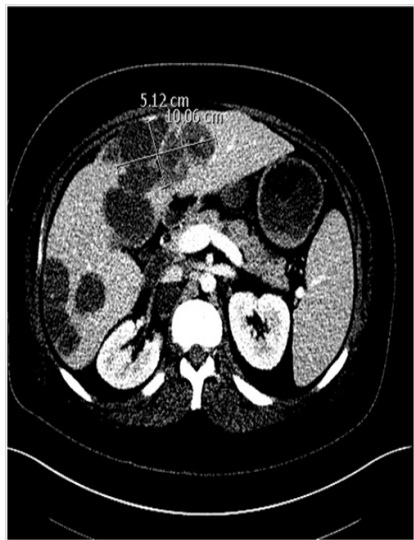

Figure IB (CT with contrast after therapy).

Table I Laboratory Data for Patient upon Arrival as well as Days I-3 of chemotherapy

\begin{tabular}{lllll}
\hline Labs & Initial labs at transfer & Day I & Day 2 & Day 3 \\
\hline AFP $(\mathrm{ng} / \mathrm{mL})$ & $>11,000$ & $>13,000$ & \\
HCG $(\mathrm{MIU} / \mathrm{mL})$ & $>175,000$ & $>225,000$ & \\
LDH $(\mathrm{U} / \mathrm{L})$ & $>10,000$ & 8869 & 8420 & 8608 \\
Alkaline Phosphatase Phosphatase Phosphatase & 650 & 650 & 643 & 744 \\
Phosphrous (mg/dL) & 6.3 & 6.6 & 8.1 & 6.6 \\
Sodium (mmol/l) & 125 & 126 & 125 & 132 \\
Potassium (mmol/l) & 4.8 & 4.6 & 5 & 4.5 \\
BUN (mg/dL) & 87 & & 66 & 74 \\
Creatinine (mg/dL) & 3.9 & 3 & 2.7 & 2.5 \\
Calcium (mg/dL) & 8.6 & 8.1 & 8.2 & 7.1 \\
ALT (U/L) & 112 & 83 & 81 & 88 \\
Uric Acid (mg/dL) & 20.3 & 11.4 & 9.9 & 7 \\
AST (U/L) & 173 & 109 & 204 & 217 \\
Total Bili (mg/dL) & 4.6 & 4.3 & 2.7 & 2.7 \\
\hline
\end{tabular}




\section{Discussion}

Germ cell tumors are the most common malignancy among men age 15-35years of age and this incidence has been rising over the past 50years. Due to the dramatic sensitivity of germ cell tumors to platinum based chemotherapy, the majority of patients even with advanced disease can be cured with cytotoxic therpy. ${ }^{7,8}$ It has been demonstrated that patients with International Germ Cell Cancer Collaborative Group (IGCCCG) poor risk non seminomatous germ cell tumors have a more tempered prognosis but still have 5year OS rates of approximately $50 \%{ }^{5,6}$ Recent trials have looked at alternative chemotherapy regimens to try to improve outcomes in poor-risk disease but since the late 1980 s, the regimen of bleomycin, etoposide, and cisplatin represents the standard of treatment for disseminated poor risk germ cell cancers. . $^{913,14}$

Our initial management in this case focused on addressing the patient's TLS with aggressive hydration, treatment of the electrolyte imbalances, and correction of the acid base disturbances. Rasburicase, a recombinant urate oxidase, was given one time immediately as it can be safely given in a patient with renal insufficiency with no need for dose adjustment. Allopurinol, a competitive xanthine oxidase inhibitor, blocks the metabolism of hypoxanthine, xanthine to uric acid, but may increase the serum xanthine and hypoxanthine levels in the serum which could lead to acute renal failure by forming xanthine crystals in the renal tubules. ${ }^{10,11}$ Therefore, dose adjustment of allopurinol was needed in this patient given his renal failure. Creatinine only improved slightly with these maneuvers and it was discussed that further derangements with initiation of cytotoxic therapy were almost certain, so hemodialysis was initiated.

The treatment of GCT typically involves cisplatin and data support the improved efficacy of cisplatin as opposed to carboplatin-based regimens in this disease. ${ }^{9}$ The standard first line regimens for poor risk NSGCT, as in this patient, are VIP and BEP but both have a cisplatin backbone (Chart 1). Given the known nephrotoxicity of cisplatin and this patient's acute need for hemodialysis, the best choice for initial chemotherapeutics was debatable.

Chart I NCCN guidelines for treatment of NSGCT.

$\begin{array}{ll}\text { EP } & \text { BEP } \\ \text { Etoposide } 100 \mathrm{mg} / \mathrm{m}^{2} \text { IV on } & \text { Etoposide } 100 \mathrm{mg} / \mathrm{m}^{2} \text { IV on Days I-5 } \\ \text { Days I-5 } & \text { Cisplatin } 20 \mathrm{mg} / \mathrm{m}^{2} \text { IV on Days I-5 } \\ \text { Cisplatin } 20 \mathrm{mg} / \mathrm{m}^{2} \text { IV on } & \text { Bleomycin 30units IV weekly on Days I, 8, and I5 or } \\ \text { Days I-5 } & \text { Days 2, 9, and I6 } \\ \text { Repeat every 2I days } & \text { Repeat every 2I days }\end{array}$

Given the rarity and complexity of this case, an extensive literature review was performed. There were two cases described in 2000 that reported spontaneous TLS in patients with GCT in which chemotherapy was started and the regimen was described. ${ }^{15}$ The first patient received single agent Etoposide $75 \mathrm{mg} / \mathrm{m}^{2}$ daily for three days, starting 2 hours after the completion of daily dialysis sessions. The patient became independent of dialysis on day 5 with steadily improving renal function. He subsequently received one course of etoposide/cisplatin and three courses of bleomycin/etoposide/cisplatin chemotherapy over the next fourteen weeks. In the second case, the patient had received dialysis for 4days (days 1-4) with improvement in biochemical abnormalities and kidney function. Single agent IV Etoposide at $50 \mathrm{mg} / \mathrm{m}^{2}$ daily was started on day 3, 2hours after completion of dialysis, and administered on days 3,5 , and 7 . The patient then received three additional courses of BEP chemotherapy over another 10 weeks.

Carboplatin is a platinum derivative that is less nephrotoxic than cisplatin and the dose can be titrated according to renal function, even in those with impaired function who require dialysis. ${ }^{16,17}$ The dose of carboplatin is generally based on body surface area and the Calvert formula, but these methods were not developed for patients with renal failure requiring hemodialysis therefore it was important to determine timing of hemodialysis. It has been shown that concentration close to the targeted AUC is achieved when hemodialysis is performed approximately $\mathrm{X}$ hours after drug administration17. After consideration of the above, the decision was made to dose the patient with carboplatin and etoposide. It was not felt to be appropriate to give cisplatin given the concern that it would cause more lasting renal failure in a patient whose TLS-induced nephropathy would hopefully

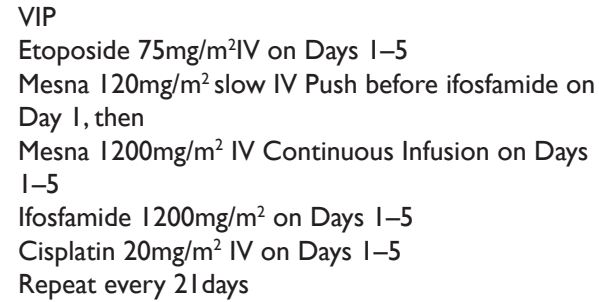

resolve with tumor control and supportive care. Carboplatin was dosed at an AUC of 5 and HD was timed at 15hours after therapy. The etoposide was given in dose attenuated fashion based on need for potential dose adjustment in the setting of renal and/or hepatic failure. ${ }^{16-18}$

This patient's clinical presentation with bulky metastatic disease, a markedly elevated serum BHCG, and a testicular mass raised the suspicion for choriocarcinoma syndrome. The patient's shortness of breath and pulmonary metastasis are also common with choriocarcinoma syndrome prompting expedited treatment as these patients are at the highest risk of morbidity and mortality from underlying disease and complications of treatment. ${ }^{20}$

The addition of bleomycin to cisplatin and etoposide improves outcomes in GCT. ${ }^{19}$ Given the acuity of this patient's presentation, his pulmonary tumor burden, as well as multi-organ dysfunction, bleomycin was excluded from the initial cycle of chemotherapy given concern for significant toxicity. ${ }^{21}$ Pulmonary toxicity is a major and potentially fatal adverse effect of this drug, with a correlation existing between creatinine clearance and the rate of bleomycin clearance from the plasma. An exponential increase in the plasma half-life of bleomycin is reported when the creatinine clearance is less than $25-35 \mathrm{ml} / \mathrm{min}^{22,23}$ Again, blemomycn was successfully added to this patient's regimen with ensuing cycles of therapy.

In summary, we present a case of spontaneous tumor lysis in a solid malignancy (prior to initiation of cytotoxic chemotherapy) and describe our method and approach to management of this critically ill patient. It is important in such a situation to expediently resolve the patient's tumor lysis and devise a chemotherapy regimen that can be 
tolerated by the patient. After the first cycle of chemotherapy, given recovery of metabolic abnormalities, a more robust regimen may be administered. ${ }^{24}$

\section{Acknowledgements}

None.

\section{Conflicts of interest}

The author declares no conflict of interest

\section{References}

1. Kanfer A, Richet G, Roland J, et al. Extreme hyperphosphataemia causing acute anuric nephrocalcinosis in lymphosarcoma. $\mathrm{Br}$ Med $\mathrm{J}$ 1979;1(6174):1320-1321.

2. Jallad B, Hamdi T, Latta S, et al. Tumor lysis syndrome in small cell lung cancer: a case report and review of the literature. Onkologie. 2011;34(3):129-131.

3. Sklarin NT and Markham M. Spontaneous recurrent tumor lysis syndrome in breast cancer. Am J Clin Oncol. 1995;18(1):71-73.

4. Cohen LF, Balow JE, Magrath IT, et al. Acute tumour lysis syndrome: review of 37 patients with Brukitt's lymphoma. Am J Med. 1980;68(4):486-491.

5. Huyyghe E, Matsuda T, Thonneau P. Increasing Incidence of testicular cancer worldwide: a review. J Urol. 2003;170(1):5-11.

6. Hanna NH, Einhorn LH. Testicular cancer-discoveries and updates. $N$ Engl J Med. 2014;371 (21):2005-2016.

7. Hannna N, Einhorn LH. Testicular cancer:a reflection on 50years of discovery. J Clin Oncol. 2014;32(28):3085-3092.

8. International Germ Cell Consensus Classification: a prognostic factorbased staging system for metastatic germ cell cancers. International germ cell cancer collaborative group. J Clin Oncol Feb. 1997;15(2):594-603.

9. Albers P, Albrecht W, Algaba F, et al. Guidelines on Testicular Cancer:2015 Update. Eur Urol. 2015;68(6):1054-1068.

10. Coiffier B, Altman A, Pui CH, et al. Guidelines for the management of pediatric and adult tumor lysis syndrome: an evidence-based review. $J$ Clin Oncol. 2008;26(16):2767-2778.

11. Hochberg J, Cairo MS. Tumor lysis syndrome: current perspective. Haematologica. 2008;93(1):9-13.
12. Xiao H, Mazumdar M, Bajorin DF, et al. Long-term follow-up of patients with good-risk germ cell tumors treated with etoposide and cisplatin. J Clin Oncol. 1997;15(7):2553-2558.

13. Saxman SB, Finch D, Gonin R, et al. Long-term follow-up of a phase III study of three versus four cycles of bleomycin, etoposide, and cisplatin in favorable-prognosis germ-cell tumors:The Indiana University Experience. J Clin Oncol. 1998;16(2):702-706.

14. Nichols CR, Catalano PJ, Crawford ED, et al. Randomized comparison of cisplatin and etoposide and either bleomycin or Ifosfamide in treatment of advanced disseminated germ cell tumors: an eastern cooperative oncology group, southwest oncology group, and cancer and leukemia group B study. J Clin Oncol. 1998;16(4):1287-1293.

15. Pentheroudakis G, O’Neli VJ, Vasey P, et al. Spontaneous acute tumor lysis syndrome in patients with metastatic germ cell tumours. Support Care Cancer. 2001;9(7):554-557.

16. Go RS, Aejei AA. Review of comparative pharmacology and clinical activity of cisplatin and carboplatin. J Clin Onocol. 1999;17(1):409-422.

17. Janus N, Thariat J, Boulanger G, et al. Proposal for dosage adjustment and timing of chemotherapy in hemodialyzed patients. Ann Oncol. 2010;21(7):1395-1403.

18. Hiraike M, Hiraki Y, Misumi N, et al. Pharmacokinetics of carboplatin in a hemodialysis patient with small-cell lung cancer. Cancer chemotherapy and Pharmacology. 2012;69(3):845-848.

19. Khurana K, Gilligan TD, Stephenson AJ. Management of poor-prognosis testicular germ cell tumors. Indian J Urol. 2010;26(1):108-114.

20. Reilley MJ, Pagliaro LC. Testicular Choriocarcinoma: a rare variant that requires a unique treatment approach. Curr Oncolo Rep. 2015;17(2):2.

21. O'Sullivan JM, Huddart RA, Norman AR, et al. Predicting the risk of bleomycin lung toxicity in patients with germ-cell tumours. Ann Oncol. 2003;14(1):91-96.

22. Kintzel PE, Dorr RT. Anticancer drug renal toxicity and elimination: dosing guidelines for altered renal function. Cancer Treat Rev. 1995;21(1):33-64.

23. Kawai K, Hinotsu S, Tomobe M, et al. Serum Cr level during chemotherapy for testicular cancer as a possible predictor of bleomycin-induced pulmonary toxicity. Jpn J Clin Oncol. 1998;28(9):546-550.

24. Bjorin DF, Sarosdy MF, Pfsiter DG, et al. Randomized trial of etoposide and cisplatin versus etoposide and carboplatin in patients with good-risk germ cell tumors:a multiinstitutional study. J Clin Oncol. 1993;11(4):598-606. 